\title{
Need for cardiac catheterisation in assessment of patients for valve surgery
}

\author{
R J C HALL, O A KADUSHI, K EVEMY \\ From the Cardiology Department, Royal Victoria Infirmary and Freeman Hospital, Newcastle upon Tyne
}

SUMMARY One hundred and six consecutive patients with valvular heart disease, in whom valve surgery was considered on clinical grounds, were studied prospectively to assess how frequently and accurately the need for valve surgery and the operation required could be specified on non-invasive grounds alone. All patients were assessed non-invasively using clinical details (history and examination), the electrocardiogram, chest $x$-ray film, and M-mode echocardiography; two dimensional echocardiography was also performed in 65 patients. On the basis of this non-invasive assessment alone the patients were assigned to group $A$ if a definite surgical recommendation, including the operation required, could be made, or group B if catheterisation was judged necessary before such a recommendation could be made. After they had been assigned to group A or B all patients were catheterised. There were 62 patients in group A; subsequent catheterisation and surgical findings confirmed that the surgical recommendation based on the non-invasive findings was correct in all patients. In 16 of these patients the surgeon was requested to operate on a specified valve or valves and also asked to inspect a further valve or valves about which there was some doubt. In only six of these patients did the subsequent catheter resolve this doubt before operation. The remaining 44 patients were placed in group B. The most frequent reason for catheterisation was doubt about severity of the valve lesion (24 patients). Such doubt was significantly more frequent in aortic or combined aortic and mitral valve then in mitral valve disease. Nine of the 24 patients had echocardiograms which were interpreted as showing that valve disease was definitely mild, and in all nine subsequent catheterisation proved this correct; therefore, the experience gained from this study suggests that catheterisation was unnecessary in these patients. The other 20 patients in group B were catheterised because of diagnostic doubt introduced by coexistent severe respiratory disease (eight patients), to assess the extent of suspected severe lesions in asymptomatic patients (four patients), or to assess suspected associated non-valvular lesions (eight patients) such as poor left ventricular function or abnormal aortic anatomy.

Therefore, catheterisation was not needed to establish the severity of valve disease or the valve operation required in $71(67 \%)$ of the patients studied (all group A and the nine patients with definite 9 echocardiographic evidence of mild disease in group B) since non-invasive assessment did this with complete accuracy.

In the last decade non-invasive cardiological investigation has developed rapidly. Despite this, many centres still regard cardiac catheterisation as an obligatory prelude to most valve surgery. Recently the need for invasive investigation of all patients before valve surgery has been questioned: first in highly selected patients with mitral valve disease ${ }^{12}$ and subsequently in a much larger series of patients with a wide variety of valve disease. ${ }^{3}$

Accepted for publication 2 December 1982
The present study was carried out prospectively $\mathrm{N}$ during the period 1978 to 1980 . It was designed to $\sigma$ examine the accuracy of recommendations for valve surgery made on clinical and non-invasive grounds $\stackrel{\varrho}{=}$ alone by comparing these recommendations with those made after cardiac catheterisation and with sur- $\stackrel{+}{-}$ gical findings.

\section{Patients and methods}

One hundred and six consecutive patients with valve 
Table 1 Final diagnosis in all 106 patients

\begin{tabular}{lcc}
\hline & Group $A$ & Group B \\
\hline Aortic stenosis & 12 & 12 \\
Aortic regurgitation & 3 & 4 \\
Aortic valve disease (mixed) & 3 & 1 \\
Mitral stenosis & 21 & 13 \\
Mitral regurgitation & 8 & 3 \\
Mitral valve disease (mixed) & 8 & 5 \\
Combined mitral and aortic disease & 7 & 6 \\
& $\frac{62}{2}$ & -44 \\
\hline
\end{tabular}

Nine patients in group A and two in group B had additional tricuspid regurgitation.

disease were studied (Table 1). Ninety nine patients were considered as possible candidates for valve surgery because of limiting symptoms (NYHA III or IV) and seven because of clinical signs suggesting very severe valve lesions despite few symptoms. Patients with previous valve replacement and acute emergencies were excluded but patients who had undergone mitral valvotomy in the past were included. During the study period four patients with acute valvular regurgitation caused by endocarditis were seen (three aortic and one aortic and mitral). All underwent successful surgery without prior catheterisation but are not included in this study.

\section{Study design}

\section{INITIAL ASSESSMENT}

All patients were evaluated by one of us $(\mathrm{RH})$ on the basis of their history, physical signs, chest $x$-ray, and echocardiogram and placed in either group A or B before cardiac catheterisation.

Group $A$ (62 patients)

In these patients it was thought that valve surgery could be recommended without prior catheterisation using only clinical and non-invasive data. Either a specific recommendation for surgery on a particular valve or valves was made (46 patients) or such a recommendation was made but the surgeon was also asked to inspect another valve about which doubt remained after non-invasive assessment (16 patients). Group B (44 patients)

In these patients catheterisation was thought to be necessary after non-invasive assessment for one of the following reasons:

(a) Doubt that the valve disease was severe enough to account for the patient's symptoms.

(b) To define precisely the severity of valve lesions in asymptomatic patients with physical signs of severe disease.

(c) To define a particular aspect which was unclear from non-invasive data but which might have altered surgical management, for example aortic root anatomy or left ventricular function.
At this stage the surgical recommendation (group A) or the recommendation for catheterisation with reasons (group B) was recorded and given to one of us (OAK). Then all patients in groups A and B were catheterised. After catheterisation patients were referred for surgery if appropriate using all available information. The precatheterisation and the postcatheterisation recommendations for surgery in group A were compared with each other and with the surgical findings. In group B the reasons for catheterisation were analysed and the catheter diagnosis and surgical findings, where available, were compared.

\section{INVESTIGATIONS}

Every patient had a posteroanterior and left lateral chest $x$-ray, 12 lead electrocardiogram, and $M$-mode echocardiogram. A two-dimensional echocardiograph machine was obtained after the beginning of the study and this investigation was performed on the last 65 patients. The severity of valve disease shown by the echocardiogram was assessed using standard criteria. ${ }^{4}$ M-mode echocardiograms were digitised when of sufficient quality ( $60 \%$ of tracings) to assess further both left ventricular function 5 and the severity of valve lesions. ${ }^{67}$ Standard catheterisation techniques were used from either the femoral or brachial approach depending on the clinical situation. Transseptal catheterisation was not used routinely. Left and right heart catheterisation was performed in 101 patients and left heart catheterisation alone in five patients with isolated aortic stenosis.

\section{CORONORY ANGIOGRAPHY (Fig. 1)}

Coronary anatomy was defined in 97 patients. In two patients it became obvious during catheterisation and before coronary angiography had been performed that valve surgery was not indicated and hence coronary angiography was not performed. The remaining seven patients in whom coronary anatomy was not defined were all under 37 years of age and did not have chest pain. The extremely high quality cineangiography possible with the equipment available (Siemens) allowed visualisation of normal or near normal coronary arteries without recourse to selective angiography in some patients without chest pain; if this failed or the patient had chest pain, selective angiography was performed. Selective coronary angiography was attempted in all patients with chest pain.

\section{Results}

GROUP A (62 patients)

Cardiac catheterisation confirmed the need for valve surgery in all 62 patients.

In 46 patients a definite surgical procedure was recommended on clinical and non-invasive grounds 


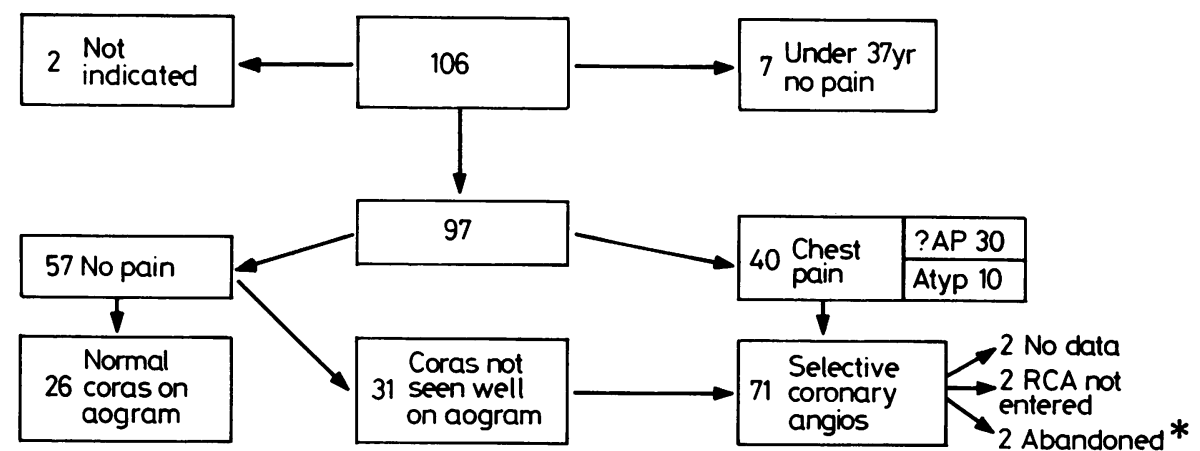

$\begin{array}{ll}* & \text { Abandoned because of prior complications } \\ \text { ?AP } & \text { Possible angina } \\ \text { Atyp } & \text { Atypical chest pain } \\ \text { Aogram } & \text { Aortogram } \\ \text { Coras } & \text { Coronary arteries }\end{array}$

Fig. 1 Investigation of coronary artery disease in all 106 patients studied.

before catheterisation. In all there was cornplete agreement between this recommendation and the catheter and surgical findings.

In 16 patients (Fig. 2) the recommendation based on precatheter data was in two parts:

(a) A definite recommendation for surgery on a particular valve or valves. In all cases this was in complete agreement with catheter and surgical findings.

(b) A second recommendation that the surgeon inspect another valve or valves and decide the need for surgery at the time of operation. Catheterisation resolved this doubt before surgery in six of the 16 patients. Doubt remained after catheterisation in 10 patients and the final recommendation before surgery still retained a request for the surgeon to inspect a valve or valves. The reasons for this uncertainty were incomplete catheter data (one patient in whom the left ventricle could not be entered and doubt remained about both the tricuspid and mitral valves), lesions of moderate severity (two patients), and doubt as to whether tricuspid regurgitation was organic or functional (seven patients).

GROUP B (44 patients)

In this group catheterisation was judged necessary to assess the severity of valvular lesions because doubt remained after clinical and non-invasive assessment ( 36 patients) or to clarify other features of possible surgical significance demonstrated inadequately by non-invasive means (eight patients). Uncertainty about the severity of valve lesions was significantly more frequent in patients with aortic or combined aortic and mitral lesions than in those with mitral valve disease alone (Table 2). Group B was divided further into four subgroups.
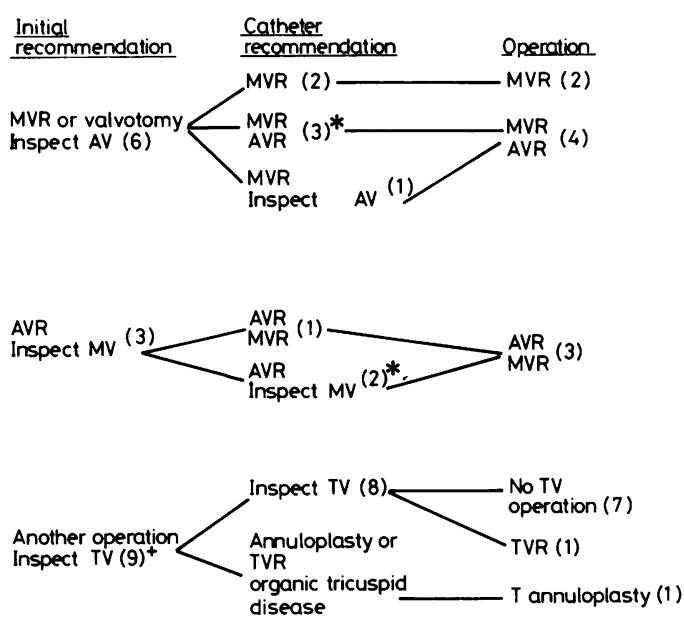

* Each group contains a patient with additional TR

+ Includes the two patients in other groups with TR MV Mitral valve

AV Aortic valve

TV Tricuspid valve

R Replacement

Fig. 2 Details of the 16 patients in whom the surgeon was requested to inspect a particular valve or valves at surgery, as well as performing a specified procedure. 
Table 2 Uncertainty in diagnosis and severity of valvular lesions in 81 patients

\begin{tabular}{|c|c|c|c|}
\hline & $\begin{array}{l}\text { Diagnosis and severity } \\
\text { certain (group } A \text { ) }\end{array}$ & $\begin{array}{l}\text { Diagnosis or severity } \\
\text { uncertain (group } B^{\star} \text { ) }\end{array}$ & Total \\
\hline $\begin{array}{l}\text { Aortic valve disease } \\
\text { Mitral valve disease } \\
\text { Combined aortic and mitral disease }\end{array}$ & $\begin{array}{r}18 \\
37 \\
7 \\
62\end{array}$ & $\begin{array}{c}13 \\
2 f \\
4 f \\
-19\end{array}$ & $\begin{array}{l}31 \\
39 \\
11 \\
81\end{array}$ \\
\hline
\end{tabular}

^Patients catheterised for reasons other than assessment of valve disease, those with definite mild disease and severe respiratory disease exçluded.

$t x^{2}$ test of frequency of uncertainty in aortic compared with mitral disease $p<0.01$ and in combined valve disease compared with mitral disease $\mathrm{p}<0.05$.

(1) Discrepancy between physical signs and/or symptoms and non-invasive investigations (24 patients)

These patients were considered according to the results of echocardiography, since this investigation gave a better guide to the true situation than any other investigation.

(a) Echocardiogram showing mild disease (nine patients) Nine patients in group B had echocardiograms interpreted as showing definitely that valve disease was mild. Six of these patients (four with mitral stenosis, one with mitral regurgitation, one with aortic stenosis) had physical signs that suggested mild disease and three had physical signs that suggested that the valve lesion might be severe (two aortic stenosis, one with aortic and mitral stenosis). In all nine, catheterisation confirmed that valve disease was mild. In retrospect these patients might have been considered as a separate group since if the echocardiogram showed unequivocally that valve disease was mild it was always correct regardless of the symptoms and physical signs. This group is small, however, and therefore further studies of this type of patient are required before catheterisation can be dispensed with in patients with significant symptoms and echocardiographically mild disease. There was one unexpected finding in this group. The patient with mild aortic and mitral stenosis was found at catheterisation to have an atrial septal defect (2:1 left to right shunt). The chest $x$-ray film and electrocardiogram were normal and the only clue to this diagnosis that had been overlooked was a moderately enlarged right ventricle ( $4 \mathrm{~cm}$ at end-diastole) on the $M$-mode echocardiogram.

\section{(b) Echocardiogram inconclusive (seven patients)}

In seven patients (two with aortic stenosis, one with aortic regurgitation, four with mitral stenosis) the physical signs suggested that disease might be severe while the echocardiogram suggested valve lesions of moderate severity in four or were of insufficient quality to allow assessment in three. At catheterisation two patients had mild disease (aortic stenosis in both), two had disease of moderate degree (one with aortic regurgitation, one with mitral stenosis), and three severe disease (mitral stenosis in all). Therefore patients with all degrees of valve disease may have inconclusive echocardiograms.

\section{(c) Echocardiogram suggesting severe disease (eight patients)}

In eight patients (three with aortic stenosis, one with subaortic stenosis, four with combined mitral and aortic valve disease) the physical signs were equivocal while the echocardiogram suggested that disease was severe. In four patients catheterisation confirmed severe valve lesions (one with subaortic stenosis, three with combined aortic and mitral valve disease) and in four showed lesions of only moderate severity. In these four patients the error arose because the two dimensional echocardiogram overestimated the severity of calcific aortic stenosis. Therefore patients with either moderate or severe disease may have echocardiograms suggesting severe disease, particularly if the valve lesion is calcific aortic stenosis.

\section{(2) Assessment of severity in asymptomatic patients}

There were four patients in this group, all with aortic stenosis, who were catheterised to define severity more precisely in order to aid a decision about valve replacement. All had signs and non-invasive investigations indicating severe disease which was confirmed at catheterisation. On the basis of the catheter results the aortic valve was replaced in one and operation deferred in three.

\section{(3) Severe symptoms and significant lung disease}

Eight patients had lung disease, confirmed by severely abnormal pulmonary function tests, which contributed significantly to symptoms (six with obstructive airways disease, two with pulmonary fibrosis). The valve lesion was mitral in all eight patients. Lung disease made non-invasive assessment difficult because the extent of its contribution to symptoms was uncertain, physical signs were obscured, echocardiography difficult (studies were unsatisfactory in five of the 
eight patients), and the potential risks of surgery were increased while potential benefits were diminished. Catheterisation showed severe lesions in five, a moderate valve lesion in one, and mild lesions in two patients.

\section{(4) Catheter for other reasons}

In eight patients there were other reasons for catheterisation. In two catheterisation was performed to assess aortic anatomy; in one because of a coarctation of the aorta associated with severe calcific valvar aortic stenosis and in the other because of an enormous aortic root shown by echo and chest $x$-ray film in a young man with aortic regurgitation (see below). In one patient with severe mitral and aortic disease a persistent ductus arteriosus was suspected on clinical grounds but not found at catheterisation. Four patients with mitral regurgitation were catheterised because non-invasive assessment suggested that left ventricular function was severely impaired; this was confirmed in all four by the left ventricular angiogram. Three of these four patients subsequently underwent valve replacement successfully but all obtained little or no benefit from the procedure. In one patient surgery was deferred. Therefore noninvasive diagnosis of severely impaired left ventricular function was invariably correct and catheterisation added nothing besides coronary artery anatomy.

\section{BOTH GROUPS}

\section{Complications of catheterisation}

There were three significant complications and no deaths. One patient with mitral valve disease developed supraventricular tachycardia during the catheterisation which led to a low output state and pulmonary oedema. This responded to treatment but curtailed the study. Another experienced a small cerebral embolus during the procedure without permanent sequelae. The third complication was more serious. A woman with angina and aortic and mitral valve disease of uncertain severity sustained a dissection of the left coronary artery when the artery was engaged without difficulty with a preformed catheter introduced from the right arm. This caused an extensive anterior myocardial infarction which the patient survived. Three months later aortic and mitral valve replacement was performed uneventfully and the patient is now symptom free.

\section{Unsuccessful and incomplete catheterisation data}

In eight patients catheter data were incomplete. In one small kyphotic woman no data were obtained and in another patient with aortic and mitral disease the left ventricle could not be entered. In four patients coronary angiography was unsuccessful though clinically indicated; in two neither coronary was entered and in two the right coronary could not be cannulated. Finally, in two patients referred to above coronary angiography was not performed because complications (supraventricular tachycardia and cerebral embolism) occurred before this stage of the procedure was reached.

\section{Coronary artery disease}

Thirty patients (17 in group A, 13 in group B) had either definite or possible angina (Fig. 1). Twelve of these patients had significant coronary lesions $(>70 \%$ obstruction of a major branch of a coronary artery). There was no predominance of a particular valve lesion and sex distribution was equal. All but two patients, aged 44 and 47 , were over the age of $54 \omega$ years. Ten patients (five in both groups) had atypical is chest pain and none had coronary lesions. Six patients $\infty$ (three men and three women) had clinically unsus- 은 pected coronary disease (two single vessel, three two $\rightarrow$ vessel, one three vessel). All were over the age of 57 years (age range 57 to 63 years) and all had mitral $\stackrel{0}{\widehat{c}}$ valve disease. The patient with three vessel disease also had a left main coronary artery lesion; she $\vec{\oplus}$ declined surgery and died three months after catheter- $\omega$ isation. In general, symptomatic and asymptomatic lesions were grafted at surgery though in seven patients only valve surgery was performed.

\section{Outcome}

Eighty patients were judged to require valve surgery; three of those, however, refused surgery. Seventy seven patients underwent surgery, with three early deaths, occurring because of renal failure and septicaemia, early dehiscence of a mitral valve prosthesis, and unsuspected dissecting aneurysm of the ascending aorta which could not be repaired at surgery. No operative complications attributable to undiagnosed coronary disease occurred in the 26 patients in whom the coronary arteries were assessed by aortography or in the nine patients in whom the coronary arteries were not assessed (Fig. 1). There was only one late death during the first year after surgery. Seven $\frac{7}{0}$ patients have shown no improvement after surgery and one patient needed reoperation because of an obs- N tructed mitral prosthesis (Omniscence disc valve). A N patient with significant coronary disease and severe $N$ aortic stenosis who had only aortic valve replacement continues to experience moderate angina.

\section{Discussion}

Cardiac catheterisation has made an enormous con- $\frac{0}{0}$ tribution to the development of modern cardiology $\overrightarrow{\mathbb{D}}$ and cardiac surgery. It has helped elucidate the $\frac{\mathbb{D}}{\mathbb{D}}$ pathophysiology of valve disease and before the advent of modern non-invasive techniques often pro- 
vided the only accurate method for assessing the severity of valvular lesions. In addition, it has stimulated careful correlation of clinical signs and non-invasive findings with the severity of valve disease. So important have these roles been that it is still regarded as a necessary routine investigation before valve surgery in many centres. Cardiac catheterisation, however, has intrinsic disadvantages since it is time consuming, expensive, unpleasant for the patient, and may produce significant complications (three in the present study). These disadvantages and the advent of accurate non-invasive techniques, of which echocardiography is the most important, have led to reassessment of its place in preoperative assessment. In some centres it has become the practice to dispense with catheterisation in selected patients with mitral lesions ${ }^{1}$ and use echocardiographic data to assess the patients' suitability for mitral valvotomy. ${ }^{2}$ Recently, Sutton $e t$ $a l .{ }^{3}$ have described excellent surgical results in a large series of patients with a wide variety of valve disease in which catheterisation was only performed in a minority of patients.

If cardiac catheterisation ceases to be a necessary preoperative procedure in a large proportion of patients with valve disease non-invasive assessment must be accurate so that surgery is safe. Such a noninvasive approach must not lead to unnecessary surgery with mild disease and if possible it must identify such patients without catheterisation. In addition, it must not deny surgery to those who need it by underestimating the severity of valve disease or result in significant associated lesions remaining uncorrected. Finally, the question of how vigorously clinically unsuspected coronary artery disease should be sought and whether its detection materially alters surgical outcome must be considered.

Both our study and that of Sutton et al. ${ }^{3}$ show that surgery can be undertaken safely in selected patients with all types of valve disease without recourse to catheterisation. The confirmation of non-invasive predictions by surgical finding in both studies excludes the possibility that the good surgical results are produced by unnecessary surgery in patients with mild disease.

Since Sutton et al. ${ }^{3}$ studied only patients who were submitted to surgery they were unable to comment on whether a non-invasive approach correctly identifies mild disease or denies surgery to those who need it. Our study allows us to draw conclusions on these points since we studied consecutive patients considered for surgery on clinical grounds some of whom were shown to have mild disease. There was no evidence that the non-invasive approach denies surgery to those who need it since no patient was mistakenly labelled as having mild disease. Furthermore, all nine patients definitely diagnosed as having mild disease by non-invasive means were also shown to have mild disease when catheterised. The four other patients with mild disease who required catheterisation to confirm this had either significant coexistent respiratory disease (two patients) or inconclusive or unsatisfactory echocardiograms (two patients). These results are slightly at variance with those of Alpert et al. ${ }^{8}$ who carried out a study of similar design. They found that decisions about management similar to those in the present study were correct in $\mathbf{9 7 \%}$ of patients; they underestimated, however, the severity of valve lesions in seven patients, four of whom had mitral valve disease and three of whom had aortic valve disease. Several factors may be responsible for the differences in results. Firstly we had two dimensional echocardiograms in more than half our patients whereas Alpert et al. ${ }^{8}$ had to rely only on M-mode echocardiograms. Secondly in the present study there was a low threshold for recommending catheterisation if symptoms, signs, or echocardiography disagreed or if echocardiograms were of poor quality. This caution should always prevent clinically significant errors in mitral valve disease since surgery is invariably guided by symptoms. Severe symptoms regardless of echocardiographic findings constituted an indication for catheterisation in the present study. In aortic valve disease the situation is slightly different since asymptomatic patients with severe aortic valve disease are sometimes considered for surgery. This, combined with the knowledge that the physical signs and echocardiographic findings can be misleading in patients with advanced aortic valve disease, ${ }^{9}$ led to even more caution in patients with aortic valve disease of doubtful severity and the significantly higher frequency with which catheterisation was recommended in aortic valve disease compared with mitral valve disease. The importance of insisting that definitely mild or severe disease should be shown by the echocardiogram before a recommendation could be made that catheterisation was unnecessary was shown by this study. Echocardiograms of poor quality or suggesting only moderate disease were obtained in three patients shown at catheterisation and surgery to have severe disease. Similarly the importance of disregarding echocardiograms suggesting disease out of proportion to the physical signs was also demonstrated; the two dimensional echocardiogram overestimated the severity of calcific aortic stenosis in four patients.

Sutton et al. ${ }^{3}$ argue convincingly that it is unlikely that significant associated lesions remained undetected in their study since the symptomatic results were so good; their data, however, do not allow a definite answer on this subject since some patients were lost to follow-up. In contrast, the present study with prospective assessment and catheterisation in all patients shows clearly that significant associated valve 
lesions were not overlooked in the patients in whom surgery was recommended without catheterisation. In one patient there was a clinically unsuspected lesion, a small atrial septal defect. By chance this lesion was found because the patient was catheterised to assess valve lesions that proved to be mild. The possible consequences of such a mistake are difficult to assess. The lesion might well have been detected at operation and was unlikely to have increased the risk of operation though uncorrected it might eventually have caused symptoms. In retrospect the only clue to the true diagnosis was a moderately increased right ventricular size ( $4 \mathrm{~cm}$ on the M-mode echo) which was disregarded when the non-invasive assessment was made.

Cardiac catheterisation, though often regarded as an infallible investigation, may itself be misleading or unhelpful. ${ }^{10}$ In this study we did not encounter any misleading results except in the one patient in whom a dissecting aneurysm was missed because the angiogram was misinterpreted; it was often unhelpful, however. In 10 of the 16 patients in whom valve inspection was recommended initially subsequent catheterisation did not resolve the clinical doubt. In several patients in whom moderate valve disease was suspected before catheterisation the diagnosis remained one of moderate disease after catheterisation. Eventually the decision of whether operation was necessary or not was made on other clinical grounds, such as age, severity of symptoms in relation to employment and lifestyle, and the patients' own preferences.

The significance of clinically unsuspected coronary disease is controversial and unresolved. 31112 Undoubtedly there are patients with valve disease who have clinically unsuspected coronary artery disease, which on occasions is severe. There were six such patients in the present study (8\% of patients undergoing surgery) all of whom had mitral valve disease; this is a somewhat lower incidence than reported in other series. ${ }^{13} 14$ Some coronary lesions may have been missed since aortography alone was performed in 26 patients. These patients did not have clinical evidence of coronary disease and the coronary arteries were well seen in all cases. Despite this, aortography cannot give completely reliable information about coronary lesions and hence some may have been missed in these 26 patients. None of these patients had complications that could be attributed to undiagnosed coronary disease either before, during, or after valve surgery. We found, as did Sutton et al., ${ }^{3}$ that coronary disease was much more frequent in elderly patients. The incidence below the age of 50 was $5 \%$ (two patients) but over the age of 60 it rose to $36 \%$ of all patients. Despite this, there is no evidence that preoperative detection of such disease and subsequent coronary surgery improves surgical results or long
Hall, Kadushi, Evemy term survival ${ }^{11} 15$ though there is a suggestion that
postoperative angina may be slightly more frequent if

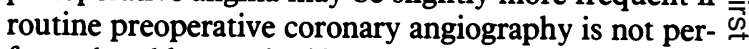
formed and hence significant, asymptomatic coronary lesions not grafted. At present it seems unreasonable $\overline{\underline{D}}$ to catheterise all patients to obtain data of doubtful $\frac{\text { के }}{5}$ significance and it remains our practice to perform $\stackrel{\unrhd}{\perp}$ coronary angiography only if there is a clinical suspi- ळ cion that coronary disease might be present and in $\overrightarrow{0}$ patients with significant risk factors, for example diabetes, heavy smokers over the age of 50 , and $\vec{\omega}$ patients with a strong family history. A recent study 16 o of patients with valve disease suggested that similar but more sophisticated risk factor analysis can predict $\overrightarrow{0}$ accurately the presence of coronary disease. If the $\omega$ cardiologist feels, on his or her assessment of available $\tilde{O}$ evidence, that coronary anatomy is essential in most $\infty$

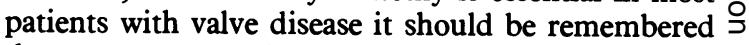
that coronary arteriography alone is a considerably $\vec{z}$ quicker and easier procedure than a full haemodynamic study for both operator and patient.

This study shows that non-invasive assessment gave a completely accurate assessment of whether cardiac $\ddot{\otimes}$ catheterisation was required before valve surgery could be recommended. In this series such an approach would have avoided catheterisation in 71 of the 106 patients studied $(67 \%)$ and 62 of the $80 \overline{0}$ patients eventually requiring surgery $(78 \%)$. This $\stackrel{0}{\circ}$ type of assessment could reduce considerably the $\stackrel{\mathbb{2}}{2}$ number of invasive procedures performed though the $\overrightarrow{\vec{O}}$ actual saving will be influenced heavily by local 3 attitudes towards asymptomatic coronary disease. Such a non-invasive approach could be incorrect if there is any disagreement between the clinical and echocardiographic findings, and depends on high quality echocardiograms interpreted by experienced cardiologists and considerable co-operation between physicians and surgeons.

\section{References}

1 Motro M, Neufeld HN. Should patients with pure mitral stenosis undergo cardiac catheterization? Am $\mathcal{F}$ Cardiol 1980; 46: 515-6.

2 Dernevik L, Brorsson L, Wallentin I, William-Olsson G. Improved results of closed commissurotomy for mitral stenosis using ultracardiography as selection ground. Acta Med Scand 1981; 210: 283-6.

3 Sutton MGStJ, Sutton MStJ, Oldershaw P, et al. Valve replacement without preoperative cardiac catheterization. $N$ Engl f Med 1981; 305: 1233-8.

4 Feigenbaum H. Echocardiography. 3rd ed. Philadelphia: Lea and Febiger, 1981.

5 Upton MT, Gibson DG. The study of left ventricular function from digitized echocardiograms. Prog Cardiovasc Dis 1978; 20: 359-84.

6 Gibson DG, Brown DJ. Measurement of instantaneous

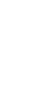


left ventricular dimension and filling rate in man, using echocardiography. Br Heart $\mathcal{F}$ 1973; 35: 1141-9.

7 Hall R, Austin A, Hunter S. M-mode echogram as a means of distinguishing between mild and severe mitral stenosis. Br Heart f 1981; 46: 486-91.

8 Alpert JS, Sloss LJ, Cohn PF, Grossman W. The diagnostic accuracy of combined clinical and non-invasive cardiac evaluation: comparison with findings at cardiac catheterization. Cathet Cardiovasc Diagn 1980; 6: 359 70.

9 Morgan RM, Hall RJC. Occult aortic stenosis as cause of intractable heart failure. $\mathrm{Br} \mathrm{Med} \mathrm{F} 1979$; i: 784-7.

10 Traill TA, Sutton MGStJ, Gibson DG. Mitral stenosis with high left ventricular diastolic pressure. Br Heart $\mathcal{F}$ 1979; 41: 405-11.

11 Bonow RO, Kent KM, Rosing DR, et al. Aortic valve replacement without myocardial revascularization in patients with combined aortic valvular and coronary artery disease. Circulation 1981; 63: 243-51.

12 Kirklin JW, Kouchoukos NT. Aortic valve replacement without myocardial revascularization. Circulation 1981; 63: 252-3.

13 Ransdale DR, Bennett DH, Bray CL, Ward C, Beton DC, Farragher FB. Coronary arteriography prior to valve replacement. Eur Heart f 1981; 2: 83-6.

14 Morrison GW, Thomas RD, Grimmer SFM, Silverton PN, Smith DR. Incidence of coronary artery disease in patients with valvular heart disease. Br Heart $\mathcal{F} 1980$; 44: 630-7.

15 Jang GG, Hancock EW. Aortic stenosis and coronary artery disease: long-term survival after aortic valve replacement (abstract). Am $\mathcal{F}$ Cardiol 1979; 43: 368.

16 Ramsdale DR, Faragher EB, Bennett DH, Bray CL, Ward C, Beton DC. Preoperative prediction of significant coronary artery disease in patients with valvular heart disease. $\mathrm{Br} \mathrm{Med} \mathcal{F} 1982 ; 284$ : 223-6.

Requests for reprints to Dr R J C Hall, Cardiology Department, Royal Victoria Infirmary, Newcastle upon Tyne NE1 4LP. 